\title{
Variation of Radiographic Protocols: An Examination of Practice During Radiography of Axial and Appendicular Skeleton in the Accra Metropolis-Ghana
}

\author{
Antwi W. K.", Gawugah J. N. K., Kyei K. A., Opoku S. Y., Arthur L., Anim Sampong S., \\ Ofori E. Batsa S.
}

Department of Radiography, School of Biomedical and Allied Health Sciences, University of Ghana, Korle-Bu, Accra, Ghana

Email address:

wkantwi@chs.edu.gh (Antwi W. K.)

\section{To cite this article:}

Antwi W. K., Gawugah J. N. K., Kyei K. A., Opoku S. Y., Arthur L., Anim Sampong S., Ofori E. Batsa S.. Variation of Radiographic Protocols: An Examination of Practice During Radiography of Axial and Appendicular Skeleton in the Accra Metropolis-Ghana. Clinical Medicine Research. Special Issue: Radiographic Practice Situation in a developing Country. Vol. 4, No. 3-1, 2015, pp. 1-4. doi: 10.11648/j.cmr.s.2015040301.11

\begin{abstract}
Background: Protocols provide a baseline for audit opportunity as well as evidence for clinical governance, since the use of protocols ensure compliance with documented scope of professional practice. Objectives: We determined the existence and variations of radiographic protocols for radiographic examinations of axial and appendicular skeleton in X-ray departments within the Accra Metropolis and also to establish whether there were variations in the projections taken among the departments for these examinations. Methods: A questionnaire containing mostly open ended question, was given to the radiographers who were in-charge of the departments and those in charge of the various clinical rooms of the selected hospitals and polyclinics to complete. Codes were assigned each of the departments, the examinations and the sets of projections indicated for the examinations. The data gathered was analyzed using SPSS version 16 software and presented in tabular and graphical forms. Results: In all eight X-ray departments were studied. Majority (80\%) of the respondents indicated having protocols. All respondents agreed to the technical fact that examination protocols must be written and documented and copies made available in all clinical rooms. Conversely, only $20 \%$ of the respondents representing $25 \%$ of the departments surveyed had examination protocols document. Conclusion: Radiographic examination protocols were lacking in most departments. There was lack of understanding among Radiographers in the departments without protocols about the concept of examination protocols and so confused it with radiographic technique. The absence of protocols led to variations in the projections taken among the departments. It also negated the radiographers' knowledge of radiographic techniques through the choice of wrong projections for some of the examinations, affecting the examination results and hence patient outcome.
\end{abstract}

Keywords: Protocols, Examination, Variation, Technique; Projections

\section{Introduction}

Right radiographic techniques and projections are vital for efficient patient management. To achieve this, radiographers and all practitioners in the field of medical imaging are required to work within agreed framework of practice termed protocol [1]. A radiographic examination protocol is an accepted and documented procedural guideline or framework which stipulates how and/or what must be done in a given situation of clinical care [1]. Protocol, in the science of medicine, is a formal set of documented rules and procedural guidelines which may be adhered to during a particular research experiment, investigation or a course of treatment
[2].

Although radiographic protocols are used as guidelines, they are however not rigid [3]. A view by Wolvekamp, [4] agrees with in indicating that protocols could be adapted to better fit special circumstances but strongly stressed on one basic rule that: "the patient's life should not be compromised or jeopardized by the radiographic examination." General radiographic technique indicates that routine radiographic examinations are carried out in two projections at right angle to each other, thus antero-posterior/postero-anterior (AP/PA) and lateral projections. These are termed basic views [4]. Basic are taken whenever an examination is requested with additional views taken only when the condition of the patient 
does not permit a basic view and/or when the diagnostic information provided by the basic views is not sufficient [5].

Schandorf \& Tetteh [6] indicate the importance of protocols in radiography practice in Ghana and identified positioning errors, improper choice of projection and wrong use of parameters such as exposure factors, source-imagedistance (SID) and screen-film combination as the main causes of film rejects. The use of effective protocols maximizes patient care and management by minimizing intra-professional variations. Protocols also allow for detailed procedural records to be kept assisting in case of legal claims [1]. Development of protocols and their use support best practice and ensure consistency across board, providing baseline for audit opportunity since their use ensure compliance with documented scope of practice [7]. It also provides common grounds for information sharing among professionals. Appropriate protocols use ensures the selection of an examination techniques and projections of high quality and accuracy, not only in terms of sensitivity (accuracy of positive findings), but also in terms of specificity (accuracy of negative findings) [4].

The use of different technique protocols and its impact on radiation dose are well documented [8]. This study performs a multi-centre investigation into the protocols used by radiographers for the same examination in the Accra metropolis

\section{Method and Materials}

The study was carried out in X-ray departments using conventional radiography departments in the selected hospitals and polyclinics both public and private in the Metropolis. The selection was done by simple ballot without replacement. Questionnaire containing mostly open-ended items was administered to radiographers who were in-charge of their departments as well as those in charge of the various clinical rooms in the lager departments to complete. Copies of the departments' protocols were also requested for verification. Data was analysed using descriptive statistics with the aid of SPSS version 16 and the results presented in tabular and graphical forms.

\section{Results}

Out of twenty-five questionnaires administered to radiographers in the eight $\mathrm{X}$-ray departments, twenty were completed and returned, representing $80 \%$ response rate. To determine the variations in the projections taken for the various examinations, the analysis was done on departmental basis. For anonymity of the study sites codes were assigned to each department, and abbreviations for the projections and examinations taken for the analysis.

\subsection{Codes for the Radiology Departments}

RD1-- --------Department in Korle-bu Teaching Hospital

RD2------ ---Department in La General Hospital

RD3--------- Department in Mamprobi Polyclinic

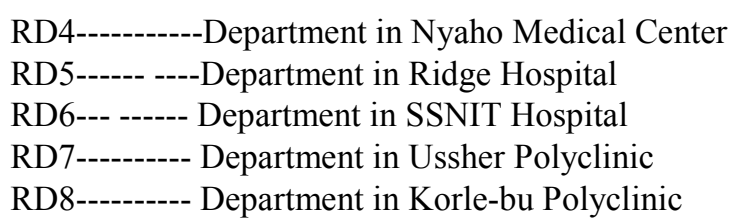

3.2. Abreviations for the Projections

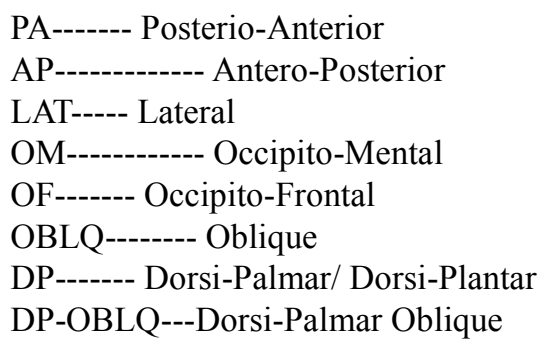

\subsection{Abreviations for the Spine}

\section{C/S---CERVICAL SPINE \\ T-L/S---THORACO-LUMBAR SPINE \\ T/S--- THORACIC SPINE \\ L-S/S---LUMBO-SACRAL SPINE \\ L/S--- LUMBAR SPINE \\ S---SACRUM}

The study showed that $80 \%$ of the respondents had radiographic protocols they work with (Figure 1) and the same indicated that these protocols were not written and documented (Figure 2). While 50\% (Table 1) of the departments use the same set of projections for skull x-ray, the rest used mixed projections for the same skull examination. With the upper limb, $87.5 \%$ of the departments use AP \& LAT for forearm; 75\% for elbow joint; $75 \%$ for humerus of the affected limb only while $25 \%$ included the unaffected humerus (Figure 3). For the lower limb, all the departments (Figure 4) take DP \& DP-OBLQ for foot; AP \& LAT for ankle joint.

For the tibia and fibula $62.5 \%$ do AP \& LAT for the affected leg and $12.5 \%$ for both legs. For the knee while $87.5 \%$ take AP \& LAT for the affected joint only, $12.5 \%$ did both knees and for femur, 75\% take AP \& LAT for the affected femur only, $12.5 \%$ examined for both femurs. The rest also vary widely on what they do. With the spine imaging (Figure 5) $57 \%$ of the departments stated AP \& LAT as the protocol in use; however $43 \%$ added the OBLQ projections for $\mathrm{C} / \mathrm{S}$. Also $86 \%$ took AP \& LAT, $14 \%$ AP, LAT \& OBLQ as the TL/S projection. $57 \%$ of the stated AP \& LAT, $43 \%$ was divided $14.3 \%$ each over projections for L-S/S.

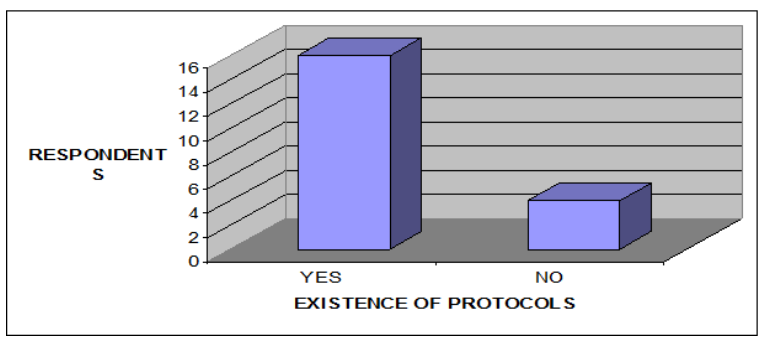

Figure 1. Existence of radiographic protocols for axial and appendicular examinations. $N=20$. 


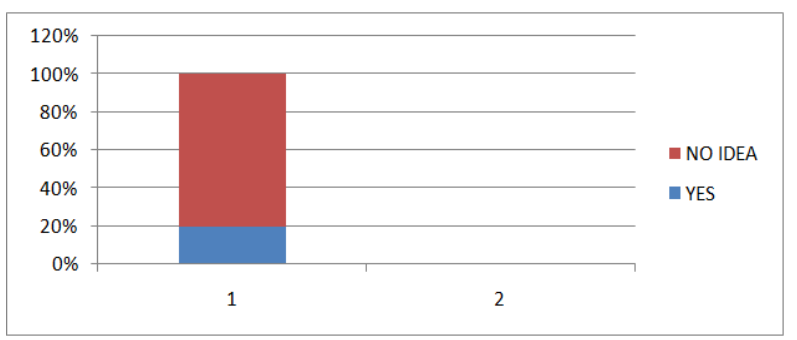

Figure 2. Existence of written and documented protocols in the departments. $N=20$.

Table 1. Projections taken by the departments for persistent headaches, skull $X$-rav. $N=8$.

\begin{tabular}{lll}
\hline Projections & Departments & Percentage \\
\hline PA \& LAT & 4 & $50 \%$ \\
LAT \& SINUSES & 1 & $12.5 \%$ \\
PA, LAT \& WALTER'S VIEW & 1 & $12.5 \%$ \\
PA, LAT \& TOWNE'S VIEW & 1 & $12.5 \%$ \\
PA, LAT \& OM & 1 & $12.5 \%$ \\
\hline
\end{tabular}

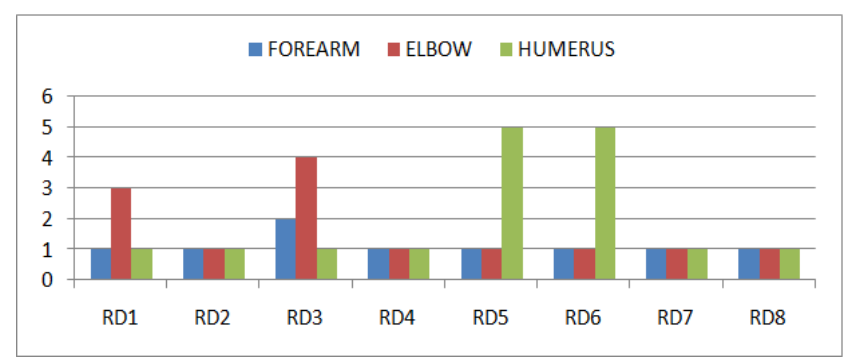

Figure 3. Projection for upper limb examinations taken among the department. $N=8$.

\subsection{Projection Codes}

AP \& LAT $=1$

LAT ONLY $=2$

AP, LAT \& OBLQ $=3$

$\mathrm{AP} \& \mathrm{AXIAL}=4$

AP \& LAT $($ BOTH LIMBS $)=5$

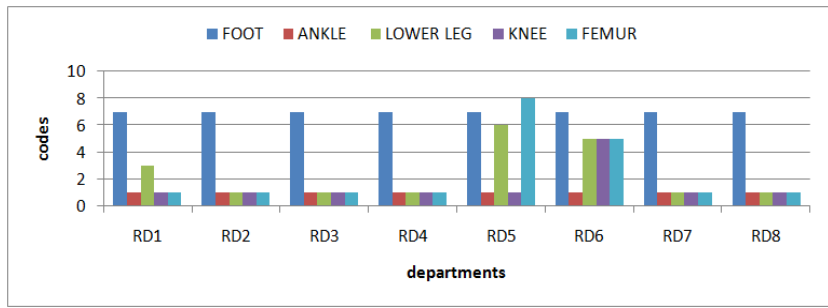

Figure 4. Projection for lower limb examinations taken by the departments. $N=8$

\subsection{Projection Codes}

AP \& $\mathrm{LAT}=1$

LAT ONLY $=2$

AP, LAT \& OBLQ $=3$

$\mathrm{AP} \& \mathrm{AXIAL}=4$

AP \& LAT $($ BOTH LIMBS $)=5$

$\mathrm{AP}$ ONLY $=6$

DP \& OBLQ $=7$

PELVIS \& OBL FUMUR $=8$

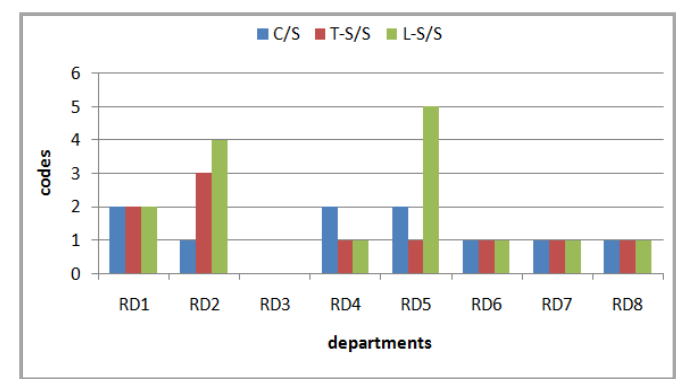

Figure 5. Projection for spine examinations taken by the departments. $N=7$.

\subsection{Codes for Spine Projections}

$$
\begin{aligned}
& \text { AP, \& LAT }=1 \\
& \text { AP, LAT, \& OBLQ }=2 \\
& \text { AP, LAT }(\text { SEPARATE })=3 \\
& \text { AP, LAT \& PELVIS }=4 \\
& \text { AP, LAT \& CONE VIEW }=5
\end{aligned}
$$

\section{Discussion}

\subsection{Existence of Protocols in the Departments}

The study determined the existence of radiographic protocols for the same radiographic examinations of axial and appendicular skeleton in X-ray departments within the Accra Metropolis and also to establish whether there were variations in the projections taken among the departments for these examinations. An examination protocol provides consistency in radiographic imaging and also reduces unnecessary exposure of patients to ionizing radiation. It was found that majority $(80 \%)$ of the respondents indicated the existence of examination protocols for axial and appendicular examinations in their departments (Figure 1).

There was also an agreement among the radiographers that examination protocols must be a written document and copies made available in all clinical rooms a concept that has been indicated in literature $[1,2]$. Conversely, it was evident that only $20 \%$ of respondents actually had protocol manuals in their department which was made available on request. This 20\% represent four respondents from two departments which constituted $25 \%$ of the eight departments studied. Thus, only $25 \%$ of the departments had protocols for axial and appendicular radiographic examinations. The respondents without protocols document in their departments however confused their knowledge of Radiography Technique from school with department protocols by arguing that the protocols existed in their head.

This suggests that if that radiographer is transferred to another hospital with existing radiographic technique protocols it may not be used regardless of what is done there. Similarly, the new person who also replaces such a radiographer with protocols in the head would have to use different technique which might not help in the management of the patients at the hospital. This implies that standard protocols would keep changing with transfers. This is not healthy for intra-professional consistency and maximizing patient care. 


\subsection{Skull and Sinuses Examinations}

Examination of the skull saw half of the departments taking the same set of projections with the remaining divided over four different sets of projections as shown in Table 1. Skull examination thus witnessed $50 \%$ variation in relation to the sets of projections taken in the departments. Sinuses examination experienced a much wider variation as only $25 \%$ indicated the same set of projections.

\subsection{Upper Limb Examinations}

All examinations involving the upper limb investigated witnessed some degrees of variation. Though seven departments $(87.5 \%)$ were found to use the same set of projections for hand with osteoarthritis, some departments did the X-ray examination for the affected hand while others examined both hands as the accepted protocols. This shows difference in ideas and techniques employed in the examinations and thus in the sensitivity of the examinations to the needs of the patient. While the both-hands (ballcatching) technique offers the opportunity for comparison between the affected and the unaffected hands, the single or affected hand technique does not. Forearm and elbow joint examinations however witnessed variations of $12.5 \%$ and $25 \%$ respectively among the departments. Humerus examination had no variation of projections among the departments but where as $75 \%$ of the departments examine only the affected arm, $25 \%$ would examine both arms for comparison increasing the radiation burden of the patient.

\subsection{Lower Limb Examinations}

All the departments agreed totally on same projections for foot and ankle joint examinations. For lower leg, knee and femur, though majority $(75 \%, 100 \%$ and $87.5 \%$ respectively) agreed on same projections, a section examine both limbs to offer comparison; while others examine only the affected leg as seen in the upper limb cases. An appreciable level of uniformity in projections taken exists among the departments in the absence of protocols. Nevertheless, the absences led to important variations where some departments take the same projections for the affected limbs alone while others take it for both limbs. It also led to only one projection being taken for a limb examination instead of two which is inadequate (Wolvekamp, 2004). Most of these variations would not exist if there were protocols in the departments since these technical principles are taken into consideration when drawing protocols.

\subsection{Spine Examinations}

Only seven departments provided information on spine examinations. Basically, all the departments indicated two important projections (AP \& LAT) in their set of projections but 3 departments included oblique views when examining the cervical spine constituting a variation among the departments. For thoraco-lumbar spine examination, $71.4 \%$ of the radiographers took the same projections for both thoracic and lumbar spine on a single film (targeting the thoraco-lumbar junction) while $14.3 \%$ also take same projection but for thoracic and lumbar spine on separate films.

The remaining $14.3 \%$ then added a different projection to that taken by the others. In examining the lumbo-sacral spine, $57 \%$ take same projections with $43 \%$ taking another. It is important to note that only one department indicated conedview (L5 - S1 junction). The coned view is an important projection because it enables good visualization of the L5-S1 joint space. Lumbo-sacral examinations over the years has revealed that most patients reporting at the department with low back pain and waist problems have narrowed L5-S1 joint space hence the importance of this projection in lumbo-sacral spine examinations.

\section{Conclusion}

It is evident that out of eight departments studied; only a small minority (two) has protocols. Radiographers in the departments without protocols really do not understand the concept of examination protocols and so confuse it with Radiographic Technique, stressing that the protocols exist in their head. So many variations exist in the projections taken among the departments for most of the examinations presented. These variations negated the radiographers' knowledge of radiographic techniques through the choice of wrong projections for some of the examinations, affecting the examination results and hence better patient outcome.

Some degrees of uniformity in projections taken were recorded among the departments in the absence of protocols. Commendable though this is, it could not however atone for the absence protocols in the departments.

\section{References}

[1] Owen A., Hogg P. and Nightingale J. (2004) A critical analysis of a locally greed protocol Radiography, 10 (2): 139-144.

[2] Bernadette S. and Cemens M. (2006) Protocol. Webster's New World Hacker's Dictionary. Indiana, USA. Wiley's Inc.

[3] The American College of Radiology. Practice guideline for general radiography. General Radiography 200815 (3): 17-21.

[4] Wolvekamp P. (2004) How to become a better Radiologist. The $29^{\text {th }}$ Congress of the World Small Animal Veterinary Association, Oct. 6-9,

[5] Sandstrom S. (2003) Radiographic Technique and Projections The WHO manual of diagnostic imaging. Lund, Sweden. WHO.

[6] Schandorf C. and Tetteh G. (1998) Analysis of the status of Xray diagnosis in Ghana. The British Journal of Radiography, 71: $1040-1048$.

[7] Professional Nursing Forum (2007) Statement of Nursing Policy \& Protocol (NP01), United Kingdom. Website: nursing policies:NP01STATEMENTOFPOLICYPROTO.doc 2004. Rhode, Greece.

[8] McEntee M.F and Kinsella C An examination of practice during radiography of the clavicle. Radiography 2010, 16 (2): $125-130$ 